\title{
Ion dynamics in perturbed quadrupole ion traps
}

\author{
G. Zs. K. Horvath, J.-L. Hernandez-Pozos, K. Dholakia, J. Rink, D. M. Segal, \\ and R. C. Thompson \\ Blackett Laboratory, Imperial College of Science, Technology and Medicine, Prince Consort Road, London SW7 2BZ, United Kingdom
}

(Received 8 August 1996; revised manuscript received 12 August 1997)

\begin{abstract}
We use the results of a Lagrangian formulation of the dynamics of ions in a Penning trap to calculate the motional frequencies of the ions as a function of the trapping parameters. We then add realistic perturbations to the ideal trapping fields in the formalism and deduce the effects of these perturbations on the ion motion. For an ideal trap there exist values of the applied trapping fields that result in a degeneracy in the ion oscillation frequencies associated with different types of motion. We show that this motional frequency degeneracy is lifted by the inclusion of the effect of a tilt of the trap axis with respect to the axis of the applied magnetic field, leading to an "avoided crossing" between the oscillation frequencies. We calculate typical ion orbits for trap parameters that give oscillation frequencies near the avoided crossing between the axial and modified cyclotron frequencies. We generalize the analysis to include the motion of ions in a combined (Penning-Paul) trap and perform an experiment to test the predictions of the theory for a degeneracy between the modified cyclotron frequency and the axial frequency for $\mathrm{Mg}^{+}$ions held in a tilted combined trap. The oscillation frequencies are measured for a range of tilt angles using a photon-photon correlation technique. There is good agreement between the experimental results and the theoretical predictions. The method we describe may prove to be a useful means by which trap imperfections can be identified and subsequently removed.
\end{abstract} [S1050-2947(98)02902-3]

PACS number(s): $32.80 . P j$

\section{INTRODUCTION}

Ions can be trapped and held at a well-localized position using a number of different schemes. The most popular schemes are based on the formation of a quadrupole electric potential using a set of three electrodes [1]. One of the electrodes (the ring) is similar to the form of the inner surface of a toroid, while the other two (end-cap) electrodes are like hemispheres above and below the plane of the ring. Ideally, the surfaces of the electrodes are hyperboloids of revolution, following the equipotential surfaces of an ideal quadrupole potential. In reality, simpler shapes are often used when the resulting small deviations from a pure quadrupole potential can be tolerated. The electrostatic quadrupole potential on its own only acts as a trap in one dimension along an axis between the end caps (the $z$ axis); the motion in the radial plane is unstable.

One method by which three-dimensional trapping may be achieved is to include a magnetic field along the $z$ axis that forces the ions into cyclotron loops if they move away from the center of the trap. The inhomogeneous electric field in the trap results in an $\mathbf{E} \times \mathbf{B}$ drift motion (magnetron motion) around the center of the trap and a shift of the cyclotron frequency (yielding the modified cyclotron frequency); see Fig. 1. This configuration, called the Penning trap, has been used for a wide variety of high-precision experiments using ions and electrons.

A completely different method for achieving threedimensional trapping is the Paul or radio-frequency trap. In this variant the magnetic field is absent and an oscillating radio-frequency potential is applied to the electrodes along with the usual dc potential. The equation of motion of ions in such a trap is a Mathieu equation and the resulting motion for a particle of given mass can be either stable or unstable depending on the applied voltages and drive frequency (unstable motion means that the ion's orbit expands rapidly until it hits one of the electrodes). The stable motion of the ions can be viewed as being made up of two components: a relatively slow harmonic oscillation about the center of the trap (the secular motion) modulated by a fast oscillation at the drive frequency (the micromotion). The micromotion amplitude increases with increasing distance from the center of the trap. A simpler, approximate mathematical treatment of the motion can be obtained by considering the oscillating potential to set up a three-dimensional pseudopotential in which the ions move. The secular motion is then just the harmonic motion of the ions in the pseudopotential. The Paul trap has also been widely used in precision experiments and spectroscopy.

A third kind of trap can be formed using the same arrangement of electrodes by applying all of the fields of the Penning and Paul traps simultaneously. The equations of mo-
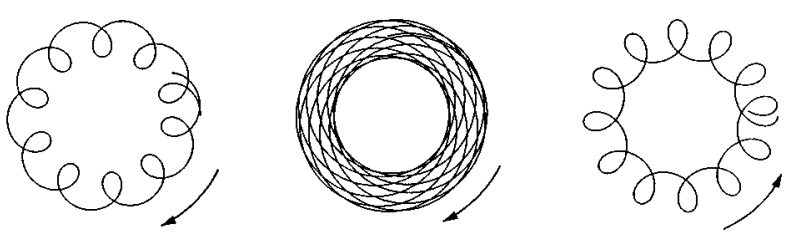

FIG. 1. Radial orbit of a single particle in quadrupole traps. (Left) Penning trap with a magnetron radius larger than the modified cyclotron radius. (Center) Same, but with the magnetron radius smaller than the modified cyclotron radius. (Right) Radial orbit in the pseudopotential approximation (the micromotion is neglected) of the combined trap (Paul side) with the amplitude of the modified cyclotron motion (clockwise) smaller than the amplitude of the magnetron motion (counter clockwise). 
tion for the resulting trap, known as the combined trap, have been derived both classically $[2,3]$ and quantum mechanically [4]. Initial experiments using this trap were performed by Fischer [2]. The motional frequencies for a combined trap have been calculated [5] and experiments have been performed to verify the calculations over a wide range of trap parameters $[5,6]$. Interest in this trap is now increasing since it has been suggested as an ideal environment in which to store simultaneously antiprotons and positrons in order to create antihydrogen [4]. The reason for its appropriateness is that it has the unique ability to store oppositely charged particles of widely differing masses in the same volume at the same time. The combined trap has been used recently to trap simultaneously ions and electrons, demonstrating this advantage [7].

The motion in a combined trap can be understood by first considering a Paul trap to which a small axial magnetic field is applied. The magnetic field has the effect of splitting the radial oscillation frequency into two components $\omega_{+}$and $\omega_{-}$ (the upper and lower radial frequencies) describing clockwise and counterclockwise rotation in the trap, respectively. As the magnetic field is increased the splitting becomes larger and $\omega_{-}$approaches zero. If the magnetic field is then held constant and the rf field is decreased, both $\omega_{+}$and $\omega_{-}$ decrease until a point is reached at which the lower frequency $\omega_{-}$becomes zero. Further decreasing the rf amplitude results in a change of sense of rotation for $\omega_{-}$such that the associated motion is now also clockwise. Finally, reducing the rf amplitude to zero results in the Penning trap with $\omega_{+}$becoming the modified cyclotron frequency and $\omega_{-}$becoming the magnetron frequency. The region of the parameter space in which $\omega_{-}$describes a rotation in an opposite sense to $\omega_{+}$is termed the "Paul side" of the combined trap. When the rf amplitude is small enough that both motions are in the same sense the combined trap is being operated on the "Penning side" (see Fig. 1).

In Ref. [5] it was shown that for particular values of the trapping parameters (applied rf voltage and magnetic field) the axial frequency $\omega_{z}$ and the upper radial frequency $\omega_{+}$ should become degenerate. Essentially this comes about because, for a fixed value of the rf potential (Paul side), increasing the magnetic field has no effect on the axial motion. The axial frequency therefore remains constant, while the upper radial frequency $\omega_{+}$increases with increasing magnetic field. Eventually, a point is reached where the two frequencies $\omega_{z}$ and $\omega_{+}$become degenerate. In Ref. [6] it was experimentally demonstrated that as the position of the calculated degeneracy is approached an "avoided crossing" develops such that the two frequencies never become exactly degenerate. A possible explanation for this behavior was put forth. It was suggested that the avoided crossing arises from a coupling between the axial motion and the radial modified cyclotron motion (in an ideal trap these motions would be completely independent). It was suggested that this coupling could arise from a misalignment of the trap symmetry axis with respect to the applied magnetic-field axis. The main purpose of this paper is twofold: (a) to present a calculation of the effect of such a misalignment and (b) to describe an experiment we have performed to verify that this misalignment is responsible for the coupling between the radial and axial motions.
We begin, in Sec. II, by describing the motion of ions in a Penning trap. The motion of a single ion has been studied in some detail by several groups $[8-10]$. We then calculate the motional frequencies of the ions as a function of the trapping parameters. In Sec. III we discuss field imperfections of various sorts concentrating in particular on the effect of a tilt between the trap axis and the magnetic-field axis. The effect of field imperfections on the motion of a single ion has been studied in the context of high-resolution mass spectroscopy by Brown and Gabrielse [8,11]. Kretzschmar has studied the effect of harmonic and anharmonic perturbations on the motion of a single ion using a perturbative approach [12]. Wineland et al. have studied the effect of some imperfections on the conservation of angular momentum of trapped particles [13]. In Sec. IV we generalize the formalism to encompass the motion in a combined trap using the pseudopotential approach. Finally, in Sec. V we describe an experiment that sets out to test some of the predictions of the theory for the motion of ions in a combined trap. In this paper we study only the motion of a single ion or the centerof-mass motion for a cloud of ions. A study of effects in clouds of ions, in particular the formation of crystals, will be presented elsewhere [14]. The calculation we will present here is exact for quadrupole perturbations, whereas the work done by Kretzschmar [12] is perturbative.

\section{MOTION OF A SINGLE ION}

We first turn our attention to the motion of a single ion in a Penning trap. In this arrangement confinement is achieved by the use of an electric-quadrupole potential, to provide confinement along the symmetry axis of the trap ( $z$ axis), with a homogeneous magnetic field along the $z$ axis providing confinement in the radial plane. For a particle of mass $m$ and charge $q$ the quadrupole electric potential is given by

$$
\Phi(x, y, z)=\frac{m \omega_{z}^{2}}{4 q}\left(2 z^{2}-x^{2}-y^{2}\right),
$$

where

$$
\omega_{z}^{2}=\frac{4 U_{0} q}{m\left(r_{0}^{2}+2 z_{0}^{2}\right)}
$$

and $U_{0}$, the potential of the end caps with respect to the ring electrode, is positive for trapping positively charged particles. We write the homogeneous magnetic field along the $z$ axis as

$$
\mathbf{B}=B \hat{\mathbf{e}}_{z}, \quad B>0 .
$$

We define the cyclotron angular frequency

$$
\omega_{c}=\frac{q B}{m} .
$$

From the Lagrangian for the system (see Ref. [12]) we obtain

$$
\begin{aligned}
& \ddot{x}=\frac{1}{2} \omega_{z}^{2} x+\omega_{c} \dot{y}, \\
& \ddot{y}=\frac{1}{2} \omega_{z}^{2} y-\omega_{c} \dot{x},
\end{aligned}
$$




$$
\ddot{z}=-\omega_{z}^{2} z
$$

Writing the radial motion in terms of the complex variable $u=x+i y$, the equation of motion in the radial plane becomes

$$
\ddot{u}=\frac{1}{2} \omega_{z}^{2} u-i \omega_{c} \dot{u} .
$$

Using the ansatz $u=e^{i \omega_{r} t}$, we obtain

$$
\omega_{r}^{2}-\omega_{c} \omega_{r}+\frac{1}{2} \omega_{z}^{2}=0,
$$

whose solutions are $\omega_{r}=-\omega_{ \pm}$with

$$
\begin{gathered}
\omega_{+}=\frac{1}{2}\left(\omega_{c}+\omega_{1}\right) \quad(\text { modified cyclotron frequency), } \\
\omega_{-}=\frac{1}{2}\left(\omega_{c}-\omega_{1}\right) \quad(\text { magnetron frequency), }
\end{gathered}
$$

where we have defined

$$
\omega_{1}^{2}=\omega_{c}^{2}-2 \omega_{z}^{2}
$$

The two frequencies $\omega_{-}$and $\omega_{+}$are positive quantities. For our configuration of fields and a positively charged particle, the circular motions associated with these frequencies of the trapped particle are clockwise (see Fig. 1). As Eq. (8) is linear, the general solution for the motion in the radial plane is given by a linear combination of the two particular solutions

$$
u(t)=r_{+} e^{-i\left(\omega_{+} t+\varphi_{+}\right)}+r_{-} e^{-i\left(\omega_{-} t+\varphi_{-}\right)},
$$

where $r_{+}, r_{-}, \varphi_{+}$, and $\varphi_{-}$are arbitrary real constants determined by the initial conditions. The motion along the axial direction is simple harmonic and is given by

$$
z(t)=r_{z} \cos \left(\omega_{z} t+\varphi_{z}\right)
$$

For typical trapping parameters the oscillation frequencies of a trapped particle satisfy $\omega_{+}>\omega_{z}>\omega_{-}$, although $\omega_{z}, \omega_{+}>\omega_{-}$is always verified. (Typical parameters for ${ }^{24} \mathrm{Mg}^{+}$ions held in our trap are $B=1 \mathrm{~T}$ and $U_{0}=10 \mathrm{~V}$, giving $\omega_{+}=2 \pi \times 572 \mathrm{kHz}, \quad \omega_{z}=2 \pi \times 274 \mathrm{kHz}, \quad \omega_{-}=2 \pi$ $\times 66 \mathrm{kHz}, \quad \omega_{c}=2 \pi \times 637 \mathrm{kHz}, \quad$ and $\omega_{1}=2 \pi \times 457 \mathrm{kHz}$.) Figure 2 shows a plot of the oscillation frequencies for ${ }^{24} \mathrm{Mg}^{+}$as a function of the electric potential $U_{0}$ for a fixed value of the magnetic field. Trapping requires that all oscillation frequencies are real. For the axial degree of freedom, this simply implies that the product $q U_{0}$ must be positive. In the radial plane, the reality condition on $\omega_{1}$, i.e., $\omega_{c}^{2}$ $>2 \omega_{z}^{2}$, gives an upper limit on the trapping potential for a given value of the magnetic field. The radial motion consists of a superposition of two clockwise circular motions: the modified cyclotron motion and the magnetron motion (the magnetron motion is simply the $\mathbf{E} \times \mathbf{B}$ drift). The result is an epicyclic orbit as plotted in Fig. 1 (left and center).

One interesting feature visible in Fig. 2 is the crossing of the axial and modified cyclotron frequencies. These two oscillation frequencies become degenerate for

$$
\omega_{z}^{\text {cross }}=\frac{2}{3} \omega_{c} .
$$

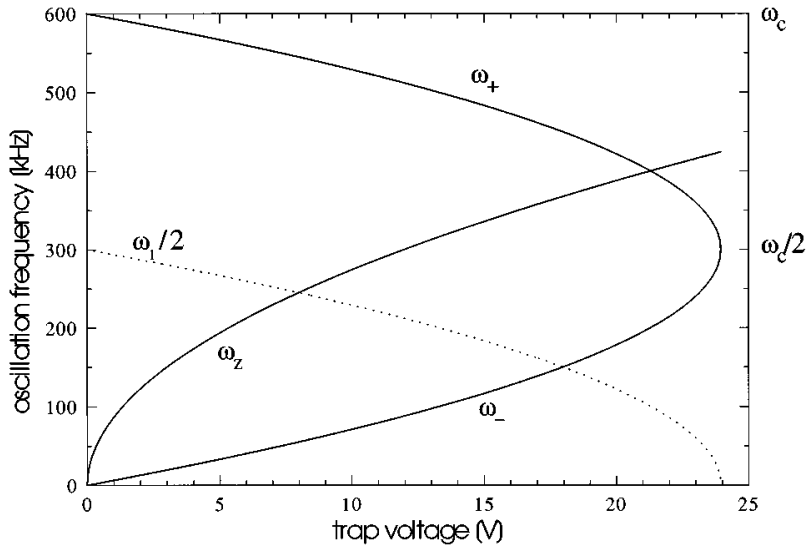

FIG. 2. Oscillation frequencies for a single ion (or the centerof-mass motion of a cloud of ions) in a Penning trap as a function of trap voltage. The frequencies are plotted for ${ }^{24} \mathrm{Mg}^{+}$ions in a trap with $R_{0}^{2}=5.40 \times 10^{-5} \mathrm{~m}^{2}$ and $B=0.9412 \mathrm{~T}$.

We will return to this feature when we discuss perturbations to the ideal trap. The other oscillation frequencies at the crossing are

$$
\begin{gathered}
\omega_{+}^{\text {cross }}=\omega_{z}^{\text {cross }}=\frac{2}{3} \omega_{c}, \\
\omega_{-}^{\text {cross }}=\frac{1}{2} \omega_{z}^{\text {cross }}=\frac{1}{3} \omega_{c} .
\end{gathered}
$$

The time-dependent Hamiltonian for a particle in this configuration of fields can be derived from the Lagrangian given in Ref. [12]. We obtain

$$
\begin{aligned}
H= & H\left(x, y, z, p_{x}, p_{y}, p_{z}\right)=\frac{1}{2 m}\left(p_{x}^{2}+P_{y}^{2}+p_{z}^{2}\right) \\
& -\frac{1}{2} \omega_{c}\left(x p_{y}-y p_{x}\right)+\frac{m}{8} \omega_{1}^{2}\left(x^{2}+y^{2}\right)+\frac{m}{2} \omega_{z}^{2} z^{2} .
\end{aligned}
$$

If we substitute the solution of the equations of motion (13) and (14) into this Hamiltonian, we obtain the single-particle Hamiltonian as a function of the radius or amplitude of the various degrees of freedom [15]

$$
H / m=\frac{1}{2} \omega_{1} \omega_{+} r_{+}^{2}-\frac{1}{2} \omega_{1} \omega_{-} r_{-}^{2}+\frac{1}{2} \omega_{z}^{2} r_{z}^{2} .
$$

Similarly, we can write the $z$ component of the canonical angular momentum (as defined in Ref. [13]) as a function of the various radii [15]

$$
l_{z}=-\frac{1}{2} m \omega_{1}\left(r_{+}^{2}-r_{-}^{2}\right) .
$$

Equations (19) and (20) deserve some comments. The Hamiltonian of Eq. (19) has nearly the form of a sum of three harmonic oscillators. Importantly, the sign of the contribution from the magnetron motion is negative. This means that if the radius of the magnetron motion is increased, the total energy decreases. Therefore, the total energy associated with the magnetron motion must be increased in order to reduce its amplitude. This fact is also clear from the canoni- 
cal angular momentum. For a stationary ion $\left(r_{+}=r_{-}=r_{z}\right.$ $=0)$, the total energy and the canonical angular momentum are zero; however, these two quantities can also individually be zero without all radii being zero, the cyclotron and axial contributions being compensated by the magnetron contribution. Therefore, contrary to a "normal" three-dimensional harmonic oscillator where the total energy has a minimum, reduction in the total energy of an ion in a Penning trap does not necessarily imply a reduction in kinetic energy.

\section{FIELD IMPERFECTIONS}

Field imperfections can be divided into two categories: harmonic and anharmonic imperfections. Imperfections of the first category will leave the motion harmonic, imperfections of the second category will render the motion nonlinear and in particular the oscillation frequencies will depend on the amplitude of the motion. Imperfections can arise from electrostatic perturbations, magnetic field inhomogeneities, and misalignment between the magnetic and the quadrupole electric fields.

Relativistic effects on the ions' motion are negligible in our case. These effects are of importance only for highresolution spectroscopic measurements; they have little effect on the dynamics of trapped clouds. For a $\mathrm{Mg}^{+}$ion in a 1 -T field, the maximum possible angular velocity is the cyclotron frequency and if we assume a maximum orbit radius of $100 \mu \mathrm{m}$ we have $\omega_{c} r=327 \mathrm{~ms}^{-1} \approx 10^{-6} c$.

Electrostatic perturbations have three origins: nonhyperbolic electrodes (truncated electrodes and access holes in the electrodes); machining errors and mounting misalignment; and contact potentials and external stray fields. The multipole expansion of the trapping field in spherical coordinates is (see, e.g., [16])

$$
\Phi(\rho, \theta, \phi)=\sum_{l, m} a_{l, m} \rho^{l} P_{l}^{m}(\cos \theta) \cos m \phi
$$

where $P_{l}^{m}$ are the associated Legendre polynomials with 0 $\leqslant m \leqslant l$.

The first term of this expansion $\Phi_{0}=a_{0,0}$ is a constant that has no physical importance. The two linear terms $\Phi_{1}$ $=a_{1,0} z+a_{1,1} x$ give rise to a constant force that would simply result in a displacement of the trap center. The quadrupole terms can be written as

$$
\Phi_{2}=\frac{1}{2} a_{2,0}\left[2 z^{2}-x^{2}-y^{2}\right]+3 a_{2,1} x z+3 a_{2,2}\left[x^{2}-y^{2}\right] .
$$

Here the first term is the perfect quadrupole potential. Addition of the second term results in a tilted quadrupole potential with respect to the $z$ axis. Finally, the third term, when added to the perfect quadrupole, results in an elliptical electric field in the radial plane. Since all three terms are quadratic, the motion remains harmonic despite the perturbation.

All higher-order terms will introduce nonlinearities in the equations of motion. By careful design, most of these terms can be made negligible. If the electrodes are symmetrical with respect to the $z=0$ plane, all $a_{l, m}$ terms with $l$ odd vanish. Cylindrical symmetry implies that $a_{l, m}=0$ for $m$ $\neq 0$. We are then left with

$$
\Phi(\rho, \theta)=\Phi_{0}+\Phi_{2}+V_{0} \sum_{l=4, \text { even }}^{\infty} c_{l}\left(\frac{\rho}{R_{0}}\right)^{l} P_{l}(\cos \theta),
$$

where $P_{l}^{0}=P_{l}$ are the usual Legendre polynomials. The dominating terms of the nonlinear contributions depend on the actual trap geometry. For example, in a trap where the ideally hyperbolic electrodes are approximated by electrodes of spherical section, the main nonlinear term is the $l=4$ term (octupole) [3]. For a cylindrical trap, the electrodes can be designed such as to minimize the octupole and then the dominating term is the $l=6$ term [17].

\section{A. Quadrupole perturbations}

\section{Constant force terms}

The effect of the linear term $\Phi_{1}$ is simply to displace the actual center of the trap. If the potential is perfectly harmonic, this has no effect on the motion of trapped ions, except for a spatial translation. However, when operating at low trap potentials the displacement can be sufficiently large to make trapping impractical [18]. The main sources of terms of this sort are contact potentials and external stray fields. The latter may arise from charge buildup on ceramic components away from the immediate trapping region. Care was taken in designing our trap to keep ceramic surfaces to a minimum and to keep any exposed ceramic surfaces far from the trapping region. We were not aware of any appreciable effects of stray external electric fields. Given our method for loading the trap (using a beam of neutral $\mathrm{Mg}$ atoms), the generation of localized contact potentials, on the other hand, was unavoidable.

\section{Elliptical electric field}

If the electric quadrupole field is not perfectly cylindrical, the dominant term will be the quadrupole contribution (22). If we assume that the field is symmetric with respect to the $x-y$ plane $\left(a_{21}=0\right)$ we obtain

$$
V(x, y, z)=\frac{1}{2} m \omega_{z}^{2}\left[z^{2}-\frac{1}{2}\left(x^{2}+y^{2}\right)+\frac{1}{2} \epsilon\left(x^{2}-y^{2}\right)\right],
$$

with

$$
\epsilon=\frac{3 a_{2,2} R_{0}^{2}}{V_{0}}
$$

In the radial plane the equipotentials are ellipses and the potential along the $z$ axis is still harmonic. The equations of motion in the radial plane are

$$
\begin{aligned}
& \ddot{x}=\frac{1}{2}(1-\epsilon) \omega_{z}^{2} x+\omega_{c} \dot{y}, \\
& \ddot{y}=\frac{1}{2}(1+\epsilon) \omega_{z}^{2} x-\omega_{c} \dot{x} .
\end{aligned}
$$

The presence of the ellipticity $\epsilon$ leads to new values of the oscillation frequencies, which for $\epsilon \ll 1$ can be shown to be

$$
\widetilde{\omega}_{ \pm} \cong \omega_{ \pm} \pm \frac{\epsilon^{2}}{8} \frac{\omega_{z}^{4}}{\omega_{ \pm} \omega_{1} \omega_{c}} .
$$

In a real quadrupole trap, we expect some electric-field ellipticity. The presence of holes in the ring electrode, ma- 
chining errors, and an inhomogeneous contact potential on the electrode surfaces are the most likely origins of a field ellipticity. It has been estimated that the presence of the two holes for the laser beam in the ring electrode of a trap similar to the one used in this work results in a value of $\epsilon$ of approximately 0.01 [19]. The presence of another two holes in the ring, which partially compensate for this effect, means that the actual value of $\epsilon$ is smaller. Machining errors are likely to be less significant. Contact potentials can cause much larger values that depend on the trap potential, $\epsilon$ increasing for smaller applied potentials. A simple calculation for a contact potential associated with a coating of magnesium on the copper electrode gives a maximum value for $\epsilon$ of about 0.05 for a potential of $10 \mathrm{~V}$, and about 0.3 when the trap potential is equal to the contact potential. Although these are maximal values, we see that we can expect the contact potential to be the dominating contribution to the electric-field ellipticity. For typical trapping parameters a value of 0.3 for $\epsilon$ is unrealistically large; nonetheless, even when $\epsilon=0.3$ we only expect a $0.07 \%$ change in $\omega_{+}$and a $5.0 \%$ change in $\omega_{-}$.

\section{Tilted quadrupole field}

As seen earlier, the addition of the $a_{2,1} x z$ term to the pure quadrupole terms results in a tilted quadrupole. The equations of motion with this quadrupole tilted in the $x-z$ plane are

$$
\begin{gathered}
\ddot{x}=+\frac{1}{2} \alpha \omega_{z}^{2} x-\frac{3}{2} \gamma \omega_{z}^{2} z+\omega_{c} \dot{y}, \\
\ddot{y}=+\frac{1}{2} \omega_{z}^{2} y-\omega_{c} \dot{x}, \\
\ddot{z}=-\frac{1}{2} \beta \omega_{z}^{2} z-\frac{3}{2} \gamma \omega_{z}^{2} x,
\end{gathered}
$$

where we have defined

$$
\begin{gathered}
\alpha=\cos ^{2} \theta-2 \sin ^{2} \theta, \quad \alpha \rightarrow 1 \quad \text { as } \theta \rightarrow 0 \\
\beta=2 \cos ^{2} \theta-\sin ^{2} \theta, \quad \beta \rightarrow 2 \text { as } \theta \rightarrow 0 \\
\gamma=\sin \theta \cos \theta, \quad \gamma \rightarrow 0 \quad \text { as } \theta \rightarrow 0 .
\end{gathered}
$$

As before, we look for solutions of the form

$$
x(t)=x_{0} e^{-i \omega t}, \quad y(t)=y_{0} e^{-i \omega t}, \quad z(t)=z_{0} e^{-i \omega t} .
$$

Substituting these into the equations of motion results in

$$
\begin{aligned}
& \left(\begin{array}{ccc}
-\omega^{2}-\frac{1}{2} \alpha \omega_{z}^{2} & i \omega \omega_{c} & \frac{3}{2} \gamma \omega_{z}^{2} \\
-i \omega \omega_{c} & -\omega^{2}-\frac{1}{2} \omega_{z}^{2} & 0 \\
\frac{3}{2} \gamma \omega_{z}^{2} & 0 & -\omega^{2}+\frac{1}{2} \beta \omega_{z}^{2}
\end{array}\right)\left(\begin{array}{l}
x_{0} \\
y_{0} \\
z_{0}
\end{array}\right) \\
& =\mathbf{M}\left(\begin{array}{l}
x_{0} \\
y_{0} \\
z_{0}
\end{array}\right)=0
\end{aligned}
$$

The oscillation frequencies are given by the solutions of the characteristic equation

$$
\begin{aligned}
\operatorname{det} \mathbf{M} & =\omega^{6}-\omega^{4} \omega_{c}^{2}-\omega^{2} \frac{1}{4} \omega_{z}^{2}\left[3 \omega_{z}^{2}-\omega_{c}^{2}-3 \omega_{c}^{2} \cos 2 \theta\right]-\frac{1}{4} \omega_{z}^{6} \\
& =0 .
\end{aligned}
$$

This equation of third order in $\omega^{2}$ has three solutions $\omega_{t}$, $\omega_{b}$ (where $t$ and $b$ signify "top" and 'bottom"), and $\widetilde{\omega}_{-}$, whose exact expressions are too complicated to be displayed here, but can easily be computed using a mathematical software package. The most interesting result is the (expected) removal of degeneracy at the crossing between the modified cyclotron and axial frequencies. As is clearly visible on Fig. 3 , the coupling between the radial and the axial motions introduced by the tilt between the magnetic-field direction and the symmetry axis of the quadrupole field leads to an avoided crossing. This removal of degeneracy complicates somewhat the notation of the frequencies; we now have

$$
\begin{aligned}
& \omega_{t} \rightarrow \omega_{z}, \quad \omega_{b} \rightarrow \omega_{+}, \quad \widetilde{\omega}_{-} \rightarrow \omega_{-} \quad \text { for } \omega_{z}>\omega_{+}, \\
& \omega_{t} \rightarrow \omega_{+}, \quad \omega_{b} \rightarrow \omega_{z}, \quad \widetilde{\omega}_{-} \rightarrow \omega_{-}, \quad \text { for } \omega_{z}<\omega_{+} .
\end{aligned}
$$

For small values of the angle of tilt, an approximate expression for the oscillation frequencies at the avoided crossing can be found:

$$
\begin{aligned}
& \omega_{t}^{\mathrm{cross}} \cong \omega_{z}\left(1+\frac{3}{4} \sqrt{2} \theta\right), \\
& \omega_{b}^{\mathrm{cross}} \cong \omega_{z}\left(1-\frac{3}{4} \sqrt{2} \theta\right), \\
& \widetilde{\omega}_{-}^{\mathrm{cross}} \cong \omega_{z} \frac{1}{2}\left(1+3 \theta^{2}\right) .
\end{aligned}
$$

The oscillation frequencies of the tilted trap are shown in Fig. 3. Also plotted is the magnitude of the avoided crossing as a function of the angle of tilt. As will be shown, there is a swap between the axial and the modified cyclotron motions as the electric potential is increased across the avoided crossing. We expect a dramatic change in the orbit of two strongly coupled motions as the trap potential is swept across the avoided crossing. We now calculate the orbit of all three motions.

As for the case of the elliptical electric field, the motion will consist of an ellipse, but this time the ellipse can also take any orientation in space. The size of the ellipse is determined from the initial conditions. By symmetry we expect the ellipse axis (perpendicular to the ellipse plane) to rotate in the plane defined by the magnetic field and the electricfield symmetry axis. In addition, the orientation of the ellipse principal axes will remain parallel and perpendicular to the plane of tilt. We start with an ellipse in the $x-y$ plane with principal axes $X_{0}$ and $Y_{0}$ :

$$
\left(\begin{array}{cc}
+X_{0} & \cos {\widetilde{\omega_{ \pm}}} t \\
-Y_{0} & \sin {\widetilde{\omega_{ \pm}}} t \\
0
\end{array}\right)
$$

A rotation of this ellipse by an angle $\Theta$ in the $x-z$ plane gives

$$
\left(\begin{array}{c}
+X_{0} \sin \theta \cos \widetilde{\omega}_{ \pm} t \\
-Y_{0} \sin \widetilde{\omega}_{ \pm} t \\
+X_{0} \cos \theta \cos \widetilde{\omega}_{ \pm} t
\end{array}\right)=\left(\begin{array}{c}
+x_{0} \cos \widetilde{\omega}_{ \pm} t \\
-y_{0} \sin \widetilde{\omega}_{ \pm} t \\
+z_{0} \cos \widetilde{\omega}_{ \pm} t
\end{array}\right) .
$$



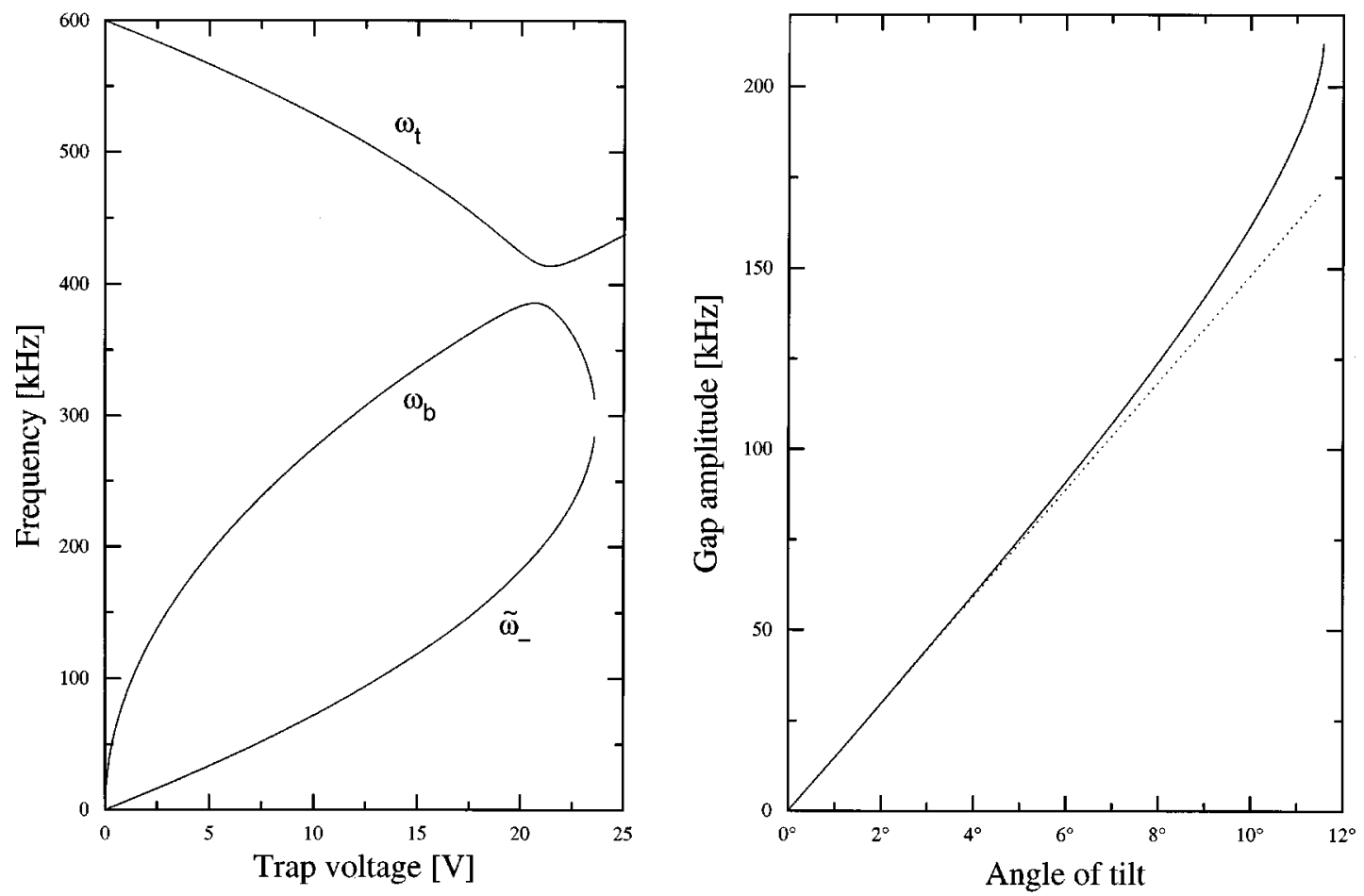

FIG. 3. (Left) Oscillation frequencies in a tilted Penning trap as a function of the trap voltage; the angle of tilt is $2^{\circ}$. (Right) Magnitude of the avoided crossing as a function of the angle of tilt. The dotted line corresponds to the linear approximation of Eq. (38).

The ellipse obtained is defined by three parameters, one to define its orientation in space $(\Theta)$, one for its aspect ratio $\left(X_{0} / Y_{0}\right)$, and finally one to determine its size $\left(X_{0}\right.$ or $\left.Y_{0}\right)$. In our case this last parameter will be defined by the initial conditions. Substituting Eq. (40) into Eq. (29) and equating the cosine and sine coefficients leads to the relations

$$
\begin{gathered}
\frac{x_{0}}{y_{0}}=-\frac{\omega^{2}+\frac{1}{2} \omega_{z}^{2}}{\omega \omega_{c}}=A(\omega), \\
\frac{x_{0}}{z_{0}}=\frac{\omega^{2}-\frac{1}{2} \beta \omega_{z}^{2}}{\frac{3}{2} \omega_{z}^{2} \gamma}=B(\omega, \theta), \\
x_{0}=y_{0} A(\omega)=Y_{0} A(\omega), \\
y_{0}=Y_{0}, \\
z_{0}=\frac{x_{0}}{B}=Y_{0} \frac{A(\omega)}{B(\omega, \theta)} .
\end{gathered}
$$

The ellipse's aspect ratio is

$$
\eta(\omega, \theta)=\frac{X_{0}}{Y_{0}}=\frac{A(\omega) \sqrt{B^{2}(\omega, \theta)+1}}{B(\omega, \theta)}
$$

and its orientation is given by

$$
\tan (\Theta)=B(\omega, \theta)
$$

where $Y_{0}$ is the initial condition that determines the size of the ellipse.

Finally the orbit of the motion of a particle is given by a linear superposition of the three eigenmodes:

$$
\begin{gathered}
x(t)=\sum_{i=t, b,-} x_{0, i} \cos \omega_{i} t=\sum_{i=t, b,-} Y_{0, i} A\left(\omega_{i}\right) \cos \omega_{i} t \\
y(t)=\sum_{i=t, b,-} y_{0, i} \sin \omega_{i} t=-\sum_{i=t, b,-} Y_{0, i} \sin \omega_{i} t, \\
z(t)=\sum_{i=t, b,-} z_{0, i} \cos \omega_{i} t=\sum_{i=t, b,-} Y_{0, i} \frac{A\left(\omega_{i}\right)}{B\left(\omega_{i}, \theta\right)} \cos \omega_{i} t .
\end{gathered}
$$

Figure 4 shows the orbit of each degree of freedom of a single ion as the electric potential is varied from well below the avoided crossing to above the crossing.

\section{Invariance theorem}

From consideration of the properties of Eq. (35) we find that

$$
\omega_{b}^{2}+\omega_{t}^{2}+\widetilde{\omega}_{-}^{2}=\omega_{c}^{2} .
$$

This equation is the invariance theorem [11]. It gives the exact cyclotron oscillation frequency in a Penning trap in terms of the measurable eigenfrequencies of an imperfect but harmonic trap. This relation is routinely used in experiments where the cyclotron frequency must be measured to high accuracy, in particular for the determination of the $g$ factor of the electron and for high-accuracy mass spectroscopy (e.g., [20]). The above analysis could be used to determine the ellipticity of the electric field and the misalignment angle. In this way the necessary correction could be made. However, as we have shown, the effects of the misalignment are strongest at the avoided crossing. By measuring the mag- 

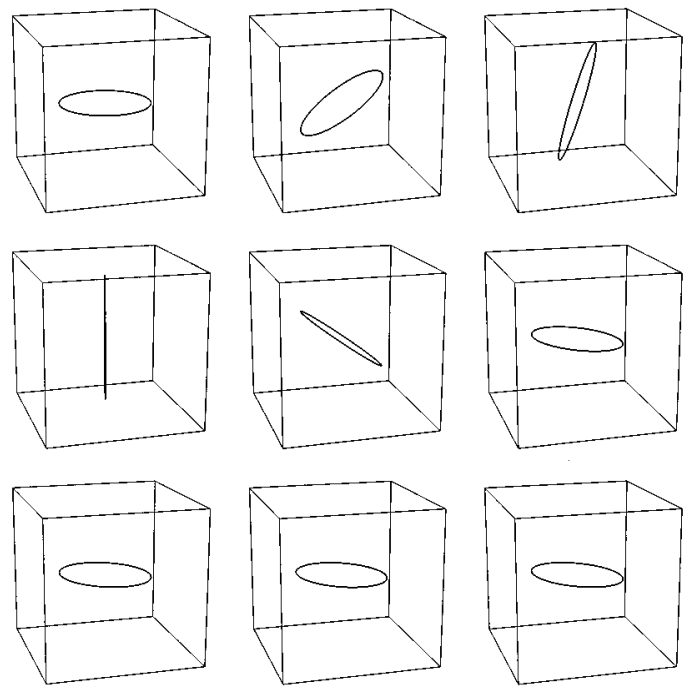

FIG. 4. Orbit in a trap whose magnetic and electric fields are at an angle of $2^{\circ}$ to each other. From top to bottom, the orbits of each degree of freedom are $\omega_{t}, \omega_{b}$, and $\omega_{-}$. The trap potential increases from left to right: below the avoided crossing (first column), at the avoided crossing (second column), and above the avoided crossing, at the stability limit (third column).

nitude of the avoided crossing the electric and magnetic fields can be accurately aligned. The accuracy of this alignment is only limited by the resolution of the measurement of the oscillation frequencies. Once the fields have been aligned, the oscillation frequencies measured away from the crossing will always be within the experimental resolution of the true oscillation frequencies if other perturbations can be neglected.

If there is no misalignment, the two frequencies become degenerate. If the electric field in the radial plane is not perfectly symmetrical, the axial frequency is unmodified but the modified cyclotron frequency is shifted to larger values [Eq. (28)]. Therefore, the crossing is moved to higher trap potentials where the relation $\omega_{-}^{\text {cross }}=\omega_{c} / 3$ does not hold. Deviations from this relation provide a means of measuring the field ellipticity and if possible, through the use of compensation electrodes, a way to compensate for it.

\section{B. Anharmonic effects}

\section{Effect of the octupole on the motion}

The octupole contribution to the electric potential can be written in the form

$$
\Phi_{4}=c_{4} \frac{V_{0}}{R_{0}^{4}}\left(8 z^{4}-24 z^{2} r^{2}+3 r^{4}\right) .
$$

If the coefficient of the quadrupole term is denoted $c_{2}$, then in a well-designed quadrupole trap, we expect to have $c_{4}$ $\ll c_{2}$, with $c_{2}$ very nearly unity. For the trap used for the experiment reported in this paper, electrodes of spherical cross section were used instead of hyperbolic ones. Bate has calculated the octupole contribution from the electrodes and has found $c_{4} \simeq 0.17$ [3]. The equations of motion cannot be solved analytically when an octupole potential is also present; however, we can perturbatively estimate the effect of this term on the motional frequencies of a single ion. This gives small frequency shifts proportional to $c_{4}$. For example, for the trap used in this work, for an orbit of radius $20 \mu \mathrm{m}$ and $c_{4} \simeq 0.17$ [3], we obtain frequency shifts of the order of $10^{-6}$, typically less than $1 \mathrm{~Hz}$.

The effect of higher-order terms can be reduced by cooling the trapped particles such that the amplitude of their motion becomes very small. The same results can be obtained by increasing the trap dimensions. As we are interested in relatively small clouds of ions, we expect the effect of nonlinearities to be much smaller than the effect of quadrupole imperfections. In particular, we expect the effect of a misalignment between the electric and magnetic fields to be more important than that due to an elliptical potential. The effects of these imperfections on small crystals formed in quadrupole traps will be presented elsewhere [14].

\section{Magnetic-field inhomogeneities}

Ideally, the magnetic field of a Penning trap is spatially homogeneous. However, it is of course impossible to create a perfectly homogeneous field. Although superconducting magnets produce much more homogeneous fields than conventional electromagnets, their inhomogeneities are still easily measurable. In the case of a conventional electromagnet, the main inhomogeneity will probably be a linear variation of the field intensity in space. For superconducting magnets, which usually have cylindrical symmetry, the dominant term is the quadrupole term (magnetic bottle).

If the magnetic field is not homogeneous then the oscillation frequency will depend on the ion's position. To a first approximation, a linear variation of the magnetic field simply leads to a broadening of the oscillation frequency linewidths. A magnetic quadrupole term will have a similar effect to the electric octupole term.

\section{THE MISALIGNED COMBINED TRAP}

In this section we calculate the oscillation frequencies of a single particle trapped in a combined trap whose electric field axis is not parallel to the direction of the magnetic field. We show that the effects of a misalignment in the combined trap are essentially the same as in the Penning trap. Since solving the "tilted" Mathieu equation exactly is probably impossible, this calculation is done using the pseudopotential approach.

\section{A. The pseudopotential of the ideal combined trap}

In the combined trap all three fields of the Paul and Penning trap are simultaneously present:

$$
\begin{aligned}
\phi(r, z) & =\frac{U_{0}+V_{0} \cos \Omega t}{R_{0}^{2}}\left(r^{2}-2 z^{2}\right) \\
& =\frac{U_{0}}{R_{0}^{2}}\left(r^{2}-2 z^{2}\right)+\frac{V_{0} \cos \Omega t}{R_{0}^{2}}\left(r^{2}-2 z^{2}\right) \\
& =\phi_{0}(r, z)+\phi_{1}(r, z) \cos \Omega t
\end{aligned}
$$

$\mathbf{B}=B \mathbf{e}_{z}$ 
A particle in a combined trap is therefore subjected to two time-independent forces and one oscillating force. It is well known that the effect of a rapidly oscillating force on a particle of mass $m$ can be approximated by the pseudopotential (see, e.g., [21])

$$
V_{\mathrm{eff}}(\mathbf{r})=\frac{\mathbf{f}(\mathbf{r}) \cdot \mathbf{f}(\mathbf{r})}{4 m \Omega}
$$

if the high frequency force is given by

$$
\mathbf{F}(\mathbf{r})=\mathbf{f}(\mathbf{r}) \cos (\Omega t)
$$

and $\mathbf{f}(\mathbf{r})$ is derivable from a potential. This result is equivalent to averaging the effect of the oscillating field over one period.

We can apply this result to the oscillating part of the electric potential. We have

$$
\mathbf{f}(\mathbf{r})=-q \nabla \phi_{1}(\mathbf{r}) .
$$

The pseudopotential is then

$$
\begin{aligned}
V_{\mathrm{osc}}(\mathbf{r}) & =\frac{m}{4}\left(\frac{2 q V_{0}}{m R_{0}^{2} \Omega}\right)^{2}\left(x^{2}+y^{2}+4 z^{2}\right) \\
& =\frac{m}{4} \omega_{d}^{2}\left(x^{2}+y^{2}+4 z^{2}\right) .
\end{aligned}
$$

The equations of motion for a single particle are then

$$
\begin{gathered}
\ddot{x}=+\frac{1}{2}\left(\omega_{s}^{2}-\omega_{d}^{2}\right) x+\omega_{c} \dot{y}, \\
\ddot{y}=+\frac{1}{2}\left(\omega_{s}^{2}-\omega_{d}^{2}\right) y-\omega_{c} \dot{x}, \\
\ddot{z}=-\left(\omega_{s}^{2}+2 \omega_{d}^{2}\right) z,
\end{gathered}
$$

where $\omega_{s}$ and $\omega_{d}$ are defined below. Substitution of solutions of the form $e^{-i \omega t}$ in these equations results in a set of three homogeneous algebraic equations. The solutions of its characteristic equation give the three oscillation frequencies

$$
\begin{gathered}
\omega_{+}=\frac{1}{2}\left(\omega_{c}+\omega_{1}\right) \quad(\text { modified cyclotron frequency }), \\
\omega_{-}=\frac{1}{2}\left(\omega_{c}-\omega_{1}\right) \quad(\text { magnetron frequency }), \\
\omega_{z}=\sqrt{\omega_{s}^{2}+2 \omega_{d}^{2}} \quad(\text { axial frequency), }
\end{gathered}
$$

where

$$
\begin{gathered}
\omega_{1}^{2}=\omega_{c}^{2}-2 \omega_{z}^{2}+\omega_{d}^{2}, \\
\omega_{d}=\frac{2 q V_{0}}{m R_{0}^{2} \Omega}, \\
\omega_{s}=\sqrt{\frac{4 q U_{0}}{m R_{0}^{2}}} \\
\omega_{c}=\frac{q B}{m} .
\end{gathered}
$$

Note that the $\omega_{1}$ defined here for the combined trap is different from that defined in Eq. (12) for the Penning trap, though they play analogous roles in the equations. If the amplitude of the oscillating field goes towards zero, one retrieves the Penning trap oscillation frequencies. Figure 5 is a plot of the combined trap oscillation frequencies calculated using the pseudopotential approach and also the exact calculation. We see that the pseudopotential calculation is essentially the same as the exact one, with the exception that the axial frequency shows significant deviation from the exact calculation as the rf amplitude increases to large values (this is not surprising as the pseudopotential approach is only strictly valid for low rf amplitude). The consequence of this is that the position of the crossing is displaced. The value of $\omega_{d}$ at which the axial and modified cyclotron frequencies become degenerate is

$$
\omega_{d}^{\text {cross }}=\frac{1}{3} \sqrt{\omega_{f}^{2}-2 \omega_{c} \omega_{f}},
$$

where

$$
\omega_{f}^{2}=4 \omega_{c}^{2}-9 \omega_{s}^{2}
$$

\section{B. The tilted combined trap}

The oscillation frequencies of the tilted combined trap can now be found in the same way as for the tilted Penning trap. For a tilted quadrupole potential the pseudopotential becomes

$$
V_{\mathrm{osc}}=\frac{m}{4} \omega_{d}^{2}\left[y^{2}+x^{2}\left(\alpha^{2}+9 \gamma^{2}\right)+z^{2}\left(\beta^{2}+9 \gamma^{2}\right)+6 \gamma x z\right],
$$

where $\alpha, \beta$ and $\gamma$ were defined in Eqs. (30)-(32). The equations of motion are

$$
\begin{gathered}
\ddot{x}=+\frac{1}{2}\left[\alpha \omega_{s}^{2}-\left(\alpha^{2}+9 \gamma^{2}\right) \omega_{d}^{2}\right] x+\frac{3}{2} \gamma\left(\omega_{s}^{2}+\omega_{d}^{2}\right) z+\omega_{c} \dot{y} \\
\ddot{y}=+\frac{1}{2}\left(\omega_{s}^{2}-\omega_{d}^{2}\right) y-\omega_{c} \dot{x} \\
\ddot{z}=-\frac{1}{2}\left[\beta \omega_{s}^{2}-\left(\beta^{2}+9 \gamma^{2}\right) \omega_{d}^{2}\right] z+\frac{3}{2} \gamma\left(\omega_{s}^{2}+\omega_{d}^{2}\right) x
\end{gathered}
$$

The oscillation frequencies are given by the characteristic equation

$$
\begin{aligned}
\omega^{6}- & \omega^{4}\left[3 \omega_{d}^{2}+\omega_{c}^{2}\right]+\omega^{2} \frac{1}{4}\left[9 \omega_{d}^{4}+\omega_{d}^{2}\left(5 \omega_{c}^{2}-6 \omega_{s}^{2}\right.\right. \\
& \left.\left.+3 \omega_{c}^{2} \cos 2 \theta\right)+\omega_{c}^{2} \omega_{s}^{2}-3 \omega_{s}^{4}+3 \omega_{c}^{2} \omega_{s}^{2} \cos 2 \theta\right]-\frac{1}{2} \omega_{d}^{6} \\
& +\frac{3}{4} \omega_{d}^{4} \omega_{s}^{2}-\frac{1}{4} \omega_{s}^{6}=0
\end{aligned}
$$

As for the Penning trap, the three solutions $\omega_{t}, \omega_{b}$, and $\widetilde{\omega}_{z}$ are too complicated to be displayed here but again can be computed easily using a mathematical software package. In the pseudopotential approximation the effect of a misalignment between the electric-field axis and the magnetic-field direction is qualitatively the same as for the Penning trap. The main feature is a removal of the degeneracy between the axial and modified cyclotron oscillation frequencies. Contrary to the situation in the Penning trap, if the confinement from the rf field is strong enough, there is no limit to the 

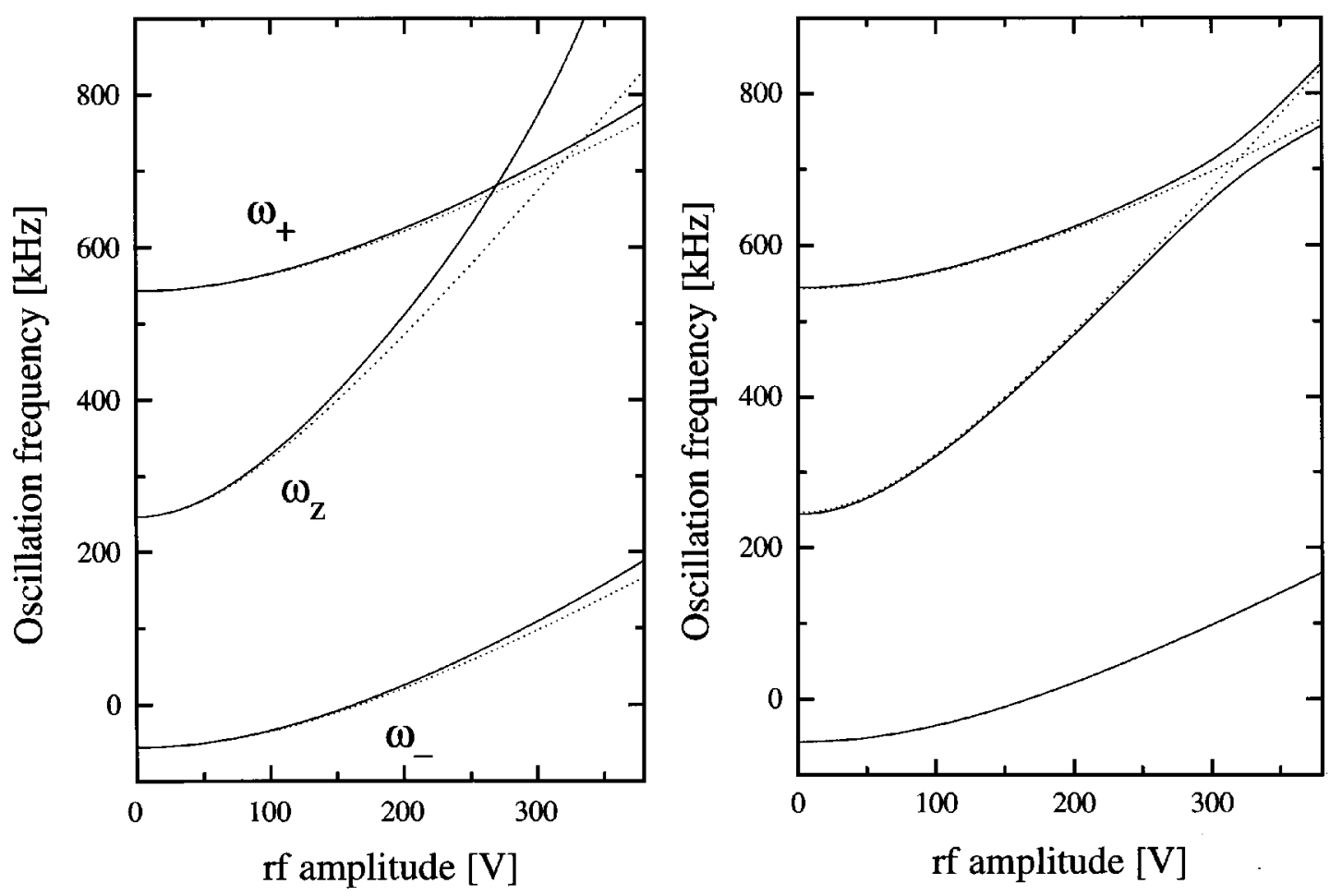

FIG. 5. Oscillation frequencies in the combined trap with a constant magnetic field. (Left) Comparison between the pseudopotential calculations (dotted lines) and exact calculations (solid lines) for a perfect trap. (Right) Oscillation frequencies in a misaligned combined trap calculated in the pseudopotential approximation. The angle of tilt between the electric-field axis and the magnetic field is $5^{\circ}$. The dotted lines give the oscillation frequencies for the ideal trap in the pseudopotential approximation.

misalignment, i.e., the motion can be stable even with the magnetic field perpendicular to the electric-field axis. $\mathrm{Nu}-$ merical investigation shows that to a first approximation the amplitude of the avoided crossing is proportional to the angle of tilt and to the amplitude of the magnetic field, the proportionality factor being a complicated function of $\omega_{s}$ and $\omega_{d}$ :

$$
G=\omega_{t}-\omega_{b} \cong F\left(\omega_{d}, \omega_{s}\right) \omega_{c} \theta, \quad \theta \ll 1,
$$

where $G$ is the size of the gap and $F\left(\omega_{d}, \omega_{s}\right)$ is some factor that depends on $\omega_{d}$ and $\omega_{s}$. This result has been obtained in the pseudopotential approximation. However, we can expect these results to be qualitatively valid even when the full effect of the rf field is taken into account.

As a part of our general investigation into the dynamics of ions held in quadrupole ion traps a comprehensive simulation program has been developed. This program computes the individual trajectories of ions in ion clouds and is capable of taking into account the effects of laser cooling, including the dependence on laser-beam position and detuning. It can also deal with the trap perturbations discussed above. We have primarily used the program to follow the evolution of the motion of small numbers of ions. Interesting effects such as crystallization can take place at the low temperatures that can be reached using laser cooling. The program has also been used to investigate the dynamics of larger clouds containing up to 50 ions. A complete description of the program and results obtained using it will be presented elsewhere [14].

The program was developed to deal with situations where a full analytical solution is not possible; however, it has also proven very useful in allowing us to cross-check the validity of our analytical results. In particular, using the simulation program, it has been checked that the amplitude of the avoided crossing gap in the "real" combined trap is indeed proportional to the angle of tilt.

\section{EXPERIMENT}

We now present an experimental verification of the presence of an avoided crossing in misaligned quadrupole traps. Our initial intention was to measure the amplitude of the avoided crossing as a function of the angle of tilt in the Penning trap. However, it was found that the trapping and laser cooling of ion clouds at high dc voltages in the Penning trap was difficult and attempts to increase the fluorescence count rate would often result in a loss of the trapped ion cloud. Horvath and Thompson [22] present a discussion of laser cooling in quadrupole ion traps in which they explore the parameter space spanned by the detuning of the cooling laser from resonance with the ion and the offset of the laser beam focus from the trap center. They demonstrate that this difficulty in operating the Penning trap at high dc voltages is expected since the cooling regions (in the space of laser beam offset vs detuning) become much smaller as the trap voltage increases. In addition, despite several attempts, even when a sufficient fluorescence count rate was obtained, no photon-photon correlation data could be observed. It was therefore decided that this experiment should be carried out in the combined trap where the photon-photon correlation 
method had been successfully used for the measurement of the oscillation frequencies and an avoided crossing had been observed [18]. The presence of the micromotion in large clouds in the combined trap limits the lowest achievable temperatures to much larger values than in the Penning trap. Since a higher cloud temperature leads to stronger modulation in the fluorescence from the cloud, we would also expect to observe more modulation in the photon-photon correlation spectrum.

\section{A. Method}

The experimental setup used has been described in detail elsewhere [6]. We use a conventional quadrupole trap with hemispherical end caps and a ring with a semicircular cross section. The internal radius of the ring is $r_{0}=5 \mathrm{~mm}$ and the end-cap spacing is $2 z_{0}=7 \mathrm{~mm}$. Two diametrically opposed holes are drilled in the ring electrode to allow the passage of the laser beam used for cooling. A further hole is drilled in the ring to allow the fluorescent light to be gathered at $90^{\circ}$ to the direction of laser propagation. In order to improve the signal-to-background ratio a blind hole is drilled into the interior surface of the ring electrode directly opposite the light gathering hole (this also partially negates the asymmetry in the potential created by the viewing hole). $\mathrm{Mg}^{+}$ions are loaded into the trap by simultaneously heating a filament, to provide electrons, and a small oven packed with Mg filings, which provides a beam of $\mathrm{Mg}$ atoms. Ions are created in situ at the center of the trap. The trap is housed in a vacuum chamber held at less than $2 \times 10^{-10}$ mbar. The vacuum chamber is located between the pole pieces of a conventional electromagnet capable of providing a magnetic field of up to $1 \mathrm{~T}$ at the minimum usable pole piece separation.

The light used for laser cooling is produced by a frequency-doubled Spectra-Physics 380D ring dye laser. The $\beta$-barium-borate frequency-doubling crystal is located in the auxiliary beam waist within the laser cavity and routinely produces $\sim 100 \mu \mathrm{W}$ of light at $280 \mathrm{~nm}$. The fluorescent light is collected using a two-lens imaging system. The first lens has a focal length of $35.1 \mathrm{~mm}$ and is held inside the vacuum chamber $28.5 \mathrm{~mm}$ from the center of the trap. A second lens outside the vacuum chamber brings the light from the ions to a focus on the photocathode of a photon-counting photomultiplier tube. A UG5 filter is placed between the second lens and the photomultiplier to discriminate against any background visible light reflected from the trap electrodes. The experiment is performed by passing the resulting photon count train to a time-to-amplitude converter followed by a multichannel analyzer. The result is a histogram of the time delays between consecutive photon counts. By taking the Fourier transform of the data produced in this way the oscillation frequencies of the ions in the trap can be measured.

In order to allow for an angle between the trap and the magnetic field, the pole pieces of the magnet were pulled slightly apart. In this way it was possible to tilt the whole vacuum chamber by as much as $10^{\circ}$ (while keeping the magnetic field at about $0.91 \mathrm{~T}$ ). In the trap region, the magnetic field is oriented parallel to the optical table. To vary the angle of tilt between the trap axis and the magnetic field axis the vacuum casing was rotated in a plane parallel to the optical table. It was assumed that the angle of misalignment in the plane perpendicular to the optical table would remain constant. Therefore, we measured only the angle in the plane parallel to the optical table $\left(\alpha_{\text {table }}\right)$. This was done by fixing a small mirror on a flat surface on the vacuum casing, with its face in a plane parallel to the magnetic field and perpendicular to the optical table. The angle of the normal to the mirror surface with the optical table edge was measured, using a helium-neon laser beam, to an accuracy of about $0.1^{\circ}$. This means that we measured the orientation of the vacuum casing and not of the trap axis. However, it can be safely assumed that the trap orientation with respect to the vacuum chamber remained constant. As a consequence, the measured angle with respect to the optical table $\left(\alpha_{\text {table }}\right)$ is related to the actual angle between the trap and the magnetic field $(\theta)$ through two constants $\left(\alpha_{0}\right.$ and $\left.\beta_{0}\right)$, such that in the limit of small angles we have

$$
\theta^{2}=\left(\alpha_{\text {table }}+\alpha_{0}\right)^{2}+\beta_{0}^{2}
$$

In order to verify the proportionality of the angle of tilt with the magnitude of the avoided crossing, the oscillation frequencies at the avoided crossing were measured for five different angles of tilt. For each angle of tilt, it was necessary to realign the detection optics and the laser-beam steering optics.

It was found to be easier to load the trap while running it in the Penning configuration than with it running as a combined trap. We therefore adopted the approach of first loading a Penning trap and then turning on the rf drive. The cloud was first laser cooled in the Penning mode until the scattering rate reached at least a few thousand counts per second. The rf amplitude was then increased in steps of approximately $10 \mathrm{~V}$. As the rf amplitude increases, the magnetron frequency decreases towards zero. At the zero magnetron frequency point, as explained by Dholakia et al. [6], the presence of a contact potential results in a displacement of the actual trap center. This displacement can be large enough to make laser cooling and even trapping impossible. This region was avoided by stepping the rf amplitude from a value slightly below the zero crossing to a value slightly above it. This corresponds to a change from the Penning side to the Paul side of the combined trap. In fact, it is rather surprising that such a "jump" could be successfully achieved since the direction of rotation of the trapped cloud changes from clockwise on the Penning side to counterclockwise on the Paul side. Indeed, such a change means that the whole cloud has to abruptly change its direction of rotation. However, if we assume that the ion motion is dominated by the magnetron motion, the cloud canonical angular momentum is relatively small (because of the small rotation frequency of the magnetron motion) and this jump corresponds in fact to a relatively small change in angular momentum. From there, the rf amplitude was increased until the avoided crossing vicinity was reached. At that point the oscillation frequencies of the cloud were measured using the photon-photon correlation technique. This was done at small intervals across the avoided crossing. 

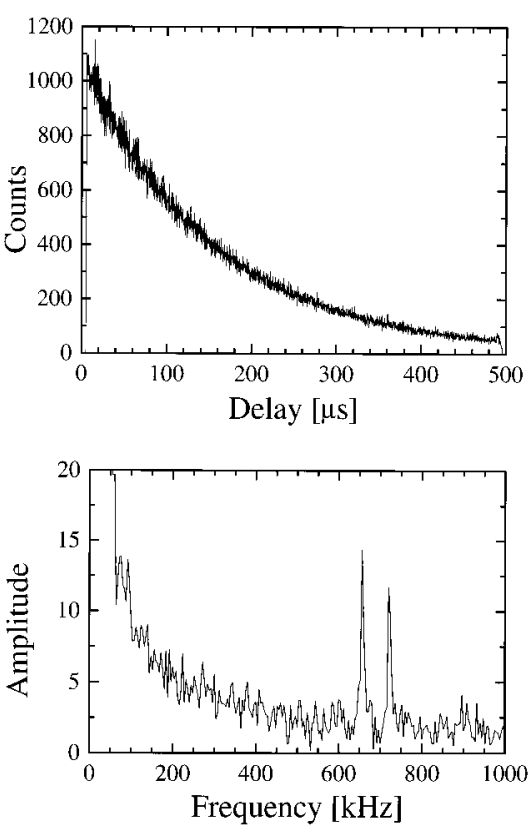

FIG. 6. (Top) Photon-photon correlation data obtained from the fluorescence of a cloud confined in a tilted combined trap. A weak modulation is clearly visible for short delays. (Bottom) Fourier transform of this photon-photon correlation data. The two peaks around $700 \mathrm{kHz}$ correspond to $\omega_{b}$ and $\omega_{t}$.

The presence of a relatively dense cloud giving a photon count rate of several thousand counts per second did not always mean that the photon-photon correlation spectrum would exhibit a modulation from which the oscillation frequencies could be obtained. In fact, quite often only components of the micromotion oscillation frequencies could be observed. In other cases, only one secular oscillation frequency was present. Both relevant oscillation frequencies (axial and modified cyclotron) were simultaneously observed usually only at their minimum separation. This is not surprising since this is when the coupling between the two degrees of freedom is strongest. When no peak appeared in the Fourier transform of the photon-photon correlation data, the laser detuning and/or the laser-beam offset were modified. When this failed, the cloud was ejected and another cloud of a different size was loaded. The real-time Fourier transform feature of our data analysis program proved to be extremely useful, as it allowed us to determine immediately whether the photon-photon correlation data contained the frequencies of interest.

It was found that, in general, medium size clouds showed a modulation in the photon-photon correlation spectrum more easily than small or large clouds. From the size of the cloud, which is estimated by scanning the laser beam across it, it is possible to estimate the number of ions to within an order of magnitude. Our "medium" clouds would typically contain between a hundred and a thousand ions.

Figure 6 shows an example of a photon-photon correlation spectrum with its Fourier transform. Note that although little modulation is visible in the photon-photon correlation data, its Fourier transform clearly shows two peaks that can be associated with $\omega_{t}$ and $\omega_{b}$.

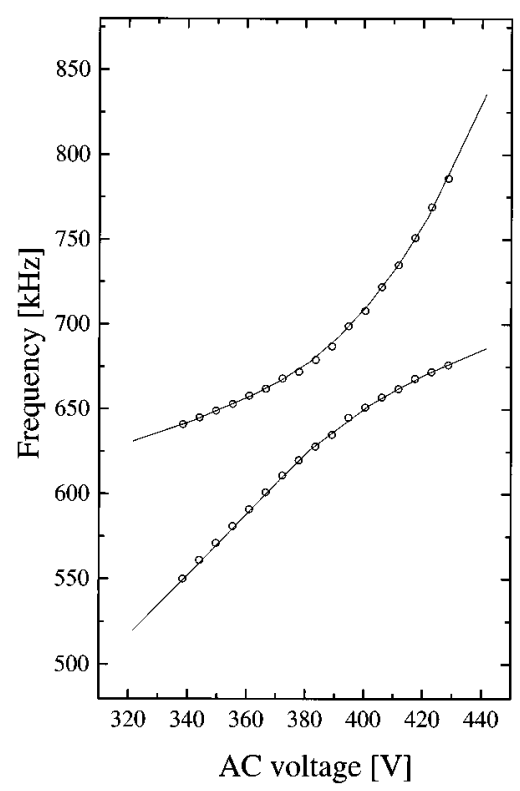

FIG. 7. Plot of the measured oscillation frequencies in the vicinity of the avoided crossing. The uncertainty in the oscillation frequencies corresponds to the size of the circles. The amplitude of the gap corresponds to an angle of tilt between the magnetic and electric fields of $4.1^{\circ} \pm 0.2^{\circ}$. The fitted solid curves are the oscillation frequencies calculated using a simulation program described in Ref. [14].

\section{B. Results and discussion}

Figure 7 shows a plot of the measured oscillation frequencies around the avoided crossing and a fit to the frequencies calculated using the simulation program. From such a plot it is possible to calculate the angle of tilt between the electric and magnetic fields. However, the proportionality factor between the angle of tilt and the amplitude of the avoided crossing depends on the actual trap parameters such as the dc and ac voltages, the magnetic field, and the geometric trap constant $R_{0}^{2}$. Measurement of the axial oscillation frequency in the Penning trap as a function of the applied dc potential provided us with a value of $R_{0}^{2}=(5.34 \pm 0.04) \times 10^{-5} \mathrm{~m}^{2}$. However, as we saw in Sec. IV, the amplitude of the avoided crossing is, to a good approximation, simply proportional to the true cyclotron frequency, which is proportional to the magnetic field. The magnetic field can be determined by fitting the data with a curve of the calculated modified cyclotron oscillation frequency for the perfect trap. Although the modified cyclotron frequency does not only depend on the magnetic field, uncertainties in $R_{0}^{2}$ and the dc and ac voltages are much smaller than the uncertainty in the magnetic field. So to first order the uncertainty in the amplitude of the avoided crossing will come from the uncertainty in the magnetic field. The proportionality factor between the angle of tilt and the amplitude of the gap [see Eq. (77)] was determined numerically using the simulation program.

The amplitude of the avoided crossing was determined by fitting a low-order polynomial to the difference between the measured values of $\omega_{t}$ and $\omega_{b}$. The amplitude of the avoided crossing was taken as the minimum of the fitted curve. In 


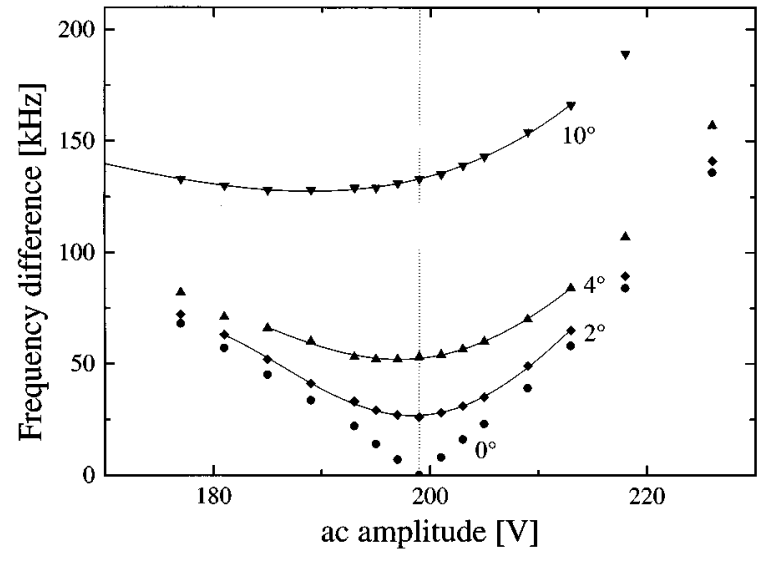

FIG. 8. Difference between $\omega_{b}$ and $\omega_{t}$ for various angles of tilt obtained numerically. For large angles the minimum separation is displaced to lower ac amplitudes.

fact, we have defined the amplitude of the gap as the difference between $\omega_{t}$ and $\omega_{b}$ taken at such trap parameters where this difference would be zero if the trap were perfect (see Fig. 8). This is not necessarily the minimum value of the difference frequency. The difference is of course due to the fact that the crossing is not symmetric. This means that the minimum we measure is not exactly the amplitude of the gap. However, we see from Fig. 8 that for small values of the angle of tilt, this error is negligible.

The angle corresponding to the avoided crossing was simply given by dividing the amplitude of the crossing by this calculated proportionality factor. The uncertainty on the angle originates from uncertainties in the magnetic field and the amplitude of the avoided crossing.

Figure 9 shows a plot of the angle of tilt calculated from the measured avoided crossing versus the measured angle of tilt of the vacuum chamber. As expected, the value of the avoided crossing does not go to zero, meaning that $\beta_{0}$ of Eq. (78) is nonzero, i.e., the trap is also misaligned in a plane perpendicular to the optical table and parallel to the magnetic field. Fitting the data points with the function given by Eq. (78) gives $\alpha_{0}=2.0^{\circ} \pm 0.1^{\circ}$ and $\beta_{0}=2.0^{\circ} \pm 0.1^{\circ}$. This assumes that $\alpha_{0}$ and $\beta_{0}$ are constant. In fact, it was observed in the latest stages of the experiment that $\beta_{0}$ could vary by up to half a degree when the angle of tilt of the trap was changed. The dotted lines in the plot of Fig. 9 correspond to the fitted curve with values of $\beta_{0}$ increased and decreased by half a degree. A $\chi^{2}$ test confirms that the fit is satisfactory.

It is clear from Fig. 9 that a misalignment between the trap electrode axis and the magnetic field results in an avoided crossing between the axial and modified cyclotron frequencies. Despite the variability of $\beta_{0}$, the data fit the theoretical curve well. If we allow for the uncertainty in $\beta_{0}$, all the data points easily fit the curve.

\section{CONCLUSIONS}

We have presented a formulation of the theory of the motion of ions in quadrupole ion traps that has allowed us to calculate the effects of common, realistic perturbations to the pure quadrupole trap. We have concentrated on the effect of a tilt between the magnetic field axis and the geometrical

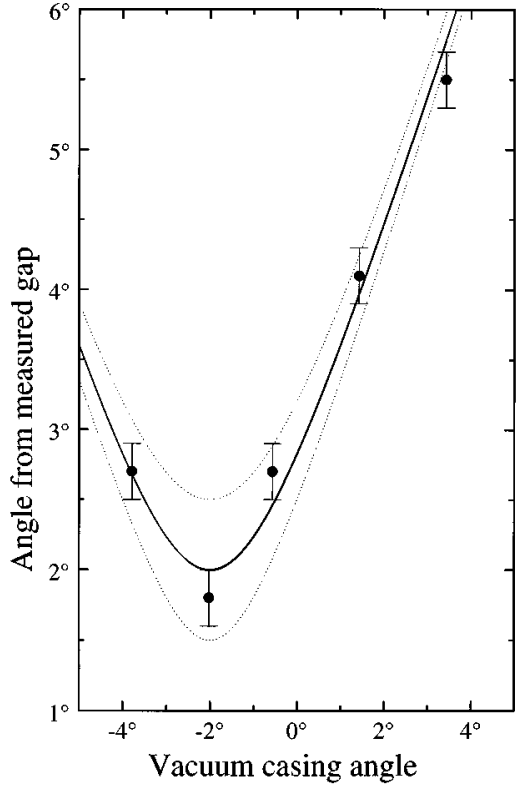

FIG. 9. Plot of the angle of tilt as calculated from the amplitude of the avoided crossing versus the angle of the vacuum casing.

axis of the trap and have performed an experiment to verify one of the predictions of the theory: that the degree to which the degeneracy between motional frequencies is lifted by the coupling introduced by the tilt is proportional to the angle of the tilt. For practical reasons we were forced to perform the experiment in a combined trap rather than in a Penning trap. Nonetheless, our experiment demonstrates a means by which a Penning or combined trap could, in principle, be accurately aligned with respect to the externally applied magnetic field. An alternative method for doing this has been demonstrated by Heinzen et al. [23]. They used two laser beams: one to cool the ions and the other to apply a torque to the ion cloud. With this arrangement they were able to control the rotation frequency of the ion cloud as a whole. In particular they were able to spin the cloud up to frequencies near to $\omega_{+}=\omega_{c}$ $-\omega_{-}$. They found that if the misalignment between the trap axis and the magnetic field axis was greater than $0.1^{\circ}$, a heating resonance was reached at a moderate rotation frequency that prevented further increase of the rotation frequency. The highest rotation frequencies (near $\omega_{c}-\omega_{-}$) were only attainable if the trap was aligned more accurately with the field. By searching for an alignment for which the heating resonance was negligible they were able to make the misalignment angle less than $0.01^{\circ}$.

\section{ACKNOWLEDGMENTS}

This work was carried out with support from the Engineering and Physical Sciences Research Council of the United Kingdom. G.Zs.K.H. would like to thank the Hélène et Victor Barbour Foundation, Geneva, the Swiss National Science Foundation, and the CVCP for financial support. JL.H-P. gratefully acknowledges the support of the Mexican Consejo Nacional de Ciencia y Tecnologia (CONACyT). J.R. gratefully acknowledges the support of the EU HCM Programme. 
[1] R. C. Thompson, Adv. At., Mol., Opt. Phys. 31, 63 (1993).

[2] E. Fischer, Z. Phys. 156, 1 (1959).

[3] D. J. Bate, Ph.D. thesis, Imperial College, London 1991 (unpublished).

[4] G.-Z. Li, Z. Phys. D 10, 451 (1988).

[5] D. J. Bate, K. Dholakia, R. C. Thompson, and D. C. Wilson, J. Mod. Opt. 39, 305 (1992).

[6] K. Dholakia, G. Zs. K. Horvath, D. M. Segal, and R. C. Thompson, J. Mod. Opt. 39, 2179 (1992).

[7] J. Walz, S. B. Ross, C. Zimmermann, L. Ricci, M. Prevedelli, and T. W. Hänsch, Phys. Rev. Lett. 75, 3257 (1995).

[8] L. S. Brown and G. Gabrielse, Rev. Mod. Phys. 58, 233 (1986).

[9] M. Kretzschmar, Z. Naturforsch. Teil A 45, 965 (1990).

[10] M. Kretzschmar, Eur. J. Phys. 12, 240 (1991).

[11] L. S. Brown and G. Gabrielse, Phys. Rev. A 25, 2423 (1982).

[12] M. Kretzschmar, Phys. Scr. 46, 544 (1992).

[13] D. J. Wineland, J. J. Bollinger, W. M. Itano, and J. D. Prestage, J. Opt. Soc. Am. B 2, 1721 (1985).
[14] G. Zs. K. Horvath and R. C. Thompson (unpublished).

[15] Wayne M. Itano and D. J. Wineland, Phys. Rev. A 25, 35 (1982).

[16] J. D. Jackson, Classical Electrodynamics (Wiley, New York, 1975).

[17] G. Gabrielse, L. Haarsma, and S. L. Rolston, Int. J. Mass Spectrom. Ion Processes 88, 319 (1989).

[18] K. Dholakia, G. Zs. K. Horvath, D. M. Segal, R. C. Thompson, D. M. Warrington, and D. C. Wilson, Phys. Rev. A 47, 441 (1993).

[19] T. Walker (private communication).

[20] F. Difilippo, V. Natarajan, K. R. Boyce, and D. E. Pritchard, Phys. Rev. Lett. 73, 1481 (1994).

[21] L. D. Landau and E. M. Lifshitz, Mechanics (Pergamon, New York, 1960).

[22] G. Zs. K. Horvath and R. C. Thompson (unpublished).

[23] D. J. Heinzen, J. J. Bollinger, F. L. Moore, Wayne M. Itano, and D. J. Wineland, Phys. Rev. Lett. 66, 2080 (1991). 